\title{
A GENERALIZED RING OF QUOTIENTS II
}

\author{
G.D. Findlay and J. Lambek
}

3. Fractional homomorphisms. A partial homomorphism $\phi$ from $B$ into A will be called a fractional homomorphism if dom $\phi \leqslant B(A)$. Every extension of a fractional homomorphism is again fractional, and each fractional homomorphism has a unique irreducible extension.

PROPOSITION 3.1. Two fractional homomorphisms have a common irreducible extension if and only if they induce the same homomorphism (necessarily fractional) on the intersection of their domains.

Proof. This follows from 1.3 (iii).

THEOREM 3.2. The irreducible fractional homomorphisms from $B$ into $A$ form an additive group $F r(B, A)$ containing Hom(B, $A)$ as a subgroup. $\operatorname{Hom}(A, A)$ and $\operatorname{Hom}(B, B)$ act as rings of left and right operators respectively.

Proof. For $\phi, \psi \in \operatorname{Fr}(B, A)$ there is a partial homomorphism $\phi+\psi$ from $B$ into $A$ defined by

$$
(\phi+\psi) b=\phi b+\psi b \quad(b \in \operatorname{dom} \phi n \operatorname{dom} \psi) \text {. }
$$

Since $\operatorname{dom} \phi \cap \operatorname{dom} \psi \leqslant B(\mathrm{~A})$ by 1.3 (iii), $\phi+\psi$ is fractional and so can be extended to a unique element of $\operatorname{Fr}(B, A)$ also denoted by $\phi+\psi$.

It is easy to verify that $\operatorname{Fr}(B, A)$ forms an additive group under + . For example, to verify the associative law, we observe that

$$
((\phi+\psi)+x) b=(\phi+(\psi+x)) b
$$

for all $b \leqslant \operatorname{dom} \phi \cap \operatorname{dom} \psi \cap \operatorname{dom} \chi \leqslant B(A)$, and hence whenever both sides of the equation are defined.

A full homomorphism of $B$ into $A$ is an irreducible partial homomorphism and is fractional by 1.1 (i). Therefore $\operatorname{Hom}(B, A)$ is a subgroup of $\operatorname{Fr}(B, A)$.

Can. Math. Bull., vol. 1, no. 3, Sept. 1958 
Finally, let $\alpha \in \operatorname{Hom}(A, A), \beta \in \operatorname{Hom}(B, B)$ and $\phi \in$ $\operatorname{Fr}(B, A)$. Then $\alpha \phi$ and $\phi \beta$ are partial homomorphisms from $B$ into $A$ with $\operatorname{dom} \alpha \phi=\operatorname{dom} \phi$ and $\operatorname{dom} \phi \beta=\beta-1 \operatorname{dom} \phi$. They are fractional since $\operatorname{dom} \phi \leqslant B(A)$ and $\beta^{-1} \operatorname{dom} \phi \leqslant B(A)$ by 1.2. They are therefore extendible to unique elements of $\operatorname{Fr}(B, A)$ also denoted by $\alpha \phi$ and $\phi \beta$. The usual associative and distributive laws follow.

PROPOSITION 3.3. If $C \leq B(A)$ then $\operatorname{Fr}(C, A) \cong \operatorname{Fr}(B, A)$.

Proof. Let $\phi \in \operatorname{Fr}(C, A)$. Then $\operatorname{dom} \phi \leq C \leq B(A)$. Hence $\phi$ can be extended to $\phi^{\prime} \in \operatorname{Fr}(B, A)$. Conversely, given such a $\phi^{\prime}$, it induces a unique $\phi$ with domain dom $\phi^{\prime} \cap \mathrm{C}$.

We remark that the isomorphism $\phi^{\prime} \rightarrow \phi$ induces the natural embedding of $\operatorname{Hom}(B, A)$ into $\operatorname{Hom}(C, A)$.

PROPOSITION 3.4. If $A \leq A^{\prime}\left(A^{\prime}\right)$ then $\operatorname{Fr}(B, A) \simeq$ $\operatorname{Fr}\left(B, A^{\prime}\right)$.

Proof. Any $\phi \in \operatorname{Fr}(\mathrm{B}, \mathrm{A})$ is also a partial homomorphism from $B$ into $A^{\prime}$. It is still fractional, since dom $\phi \leqslant B(A)$ implies dom $\phi \leqslant B\left(A^{\prime}\right)$ by 2.1 and 2.2 . Let $\phi^{\prime}$ be its irreducible extension. The mapping $\phi \rightarrow \phi^{\prime}$ is clearly an isomorphism.

To show that it is onto, let $\psi \in \operatorname{Fr}\left(B, A^{\prime}\right)$. This induces a homomorphism $\phi: \psi^{-1} \mathrm{~A} \rightarrow$ A by restricting its domain. Clearly, $\phi$ is an irreducible partial homomorphism from B into A. Moreover, it is fractional, since $\operatorname{dom} \phi=\psi^{-1} \mathrm{~A} \leqslant \operatorname{dom} \psi$ $\leq B\left(A^{\prime}\right)$ by 1.2 and 1.3 (ii) and therefore dom $\phi \leqslant B(A)$ by 1.1 (ii). Thus $\phi^{\prime}=\psi$ and so the mapping under consideration is onto.

4. Rational completeness. The module A will be called rationally complete if every fractional homomorphism from any module $B$ into $A$ is extendible to a full homomorphism.

Thus $A$ is rationally complete if and only if $\operatorname{Fr}(B, A)=$ $\operatorname{Hom}(B, A)$ for all $B$.

THEOREM 4.1. A is rationally complete if and only if A has no proper rational extension.

Proof. Assume $A$ is rationally complete and let $A^{\prime}$ be a rational extension of $A$. Then $A \leq A^{\prime}(A)$ by 2.2. Thus the 
identity mapping of $A$ is a fractional homomorphism from $A^{\prime}$ onto $A$ and hence can be extended to a full homomorphism of $A^{\prime}$ onto A. This is a homomorphism over $\AA$ and hence an isomorphism by 2.4. Therefore $\mathrm{A}^{\mathrm{i}}=\mathrm{A}$.

Conversely, assume that $A$ has no proper rational extension. We wish to show that any $\phi \in \operatorname{Fr}(B, A)$ is a full homomorphism. Its graph $G(\phi)=\{(b, \phi \bar{b}) \mid$ bedom $\phi\}$ is a submodule of the direct sum $B+A$. The projection $\pi$ of $B+A$ onto $B+A-G(\phi)=A(\phi)$ induces an isomorphism between $A$ and $\pi A$, because $\operatorname{An} G(\phi)=0$. Thus we may identify $A$ with $\pi$ A and regard $A(\phi)$ as an extension of $A$ so that $\pi b=\pi(-\phi b)=-\phi b$ for any $\mathrm{b} \in \operatorname{dom} \phi$. If $\phi^{\prime}: \mathrm{B} \rightarrow \mathrm{A}(\phi)$ is defined by $\phi^{\prime} \mathrm{b}=-\pi \mathrm{b}$ this shows that $\phi$ and $\phi^{\prime}$ coincide on dom $\phi \subseteq$ dom $\phi^{\prime}$ and so $\phi^{\prime}$ extends $\phi$. We shall show that $A(\phi)$ is a rational extension of $A$ and therefore coincides with $A$. Hence $\phi$ (which is given to be an irreducible partial homomorphism into A) coincides with $\phi^{\prime}$ and so is a full homomorphism, as was to be proved.

In view of 2.2 , we need to show that $A(\phi)$ is an essential extension of $A$ and that $A \leqslant A(\phi)(A)$.

Let $\alpha$ be a partial homomorphism from $A(\phi)$ into $A$ extending the identity mapping of $A$. It will follow that $A(\phi)$ is an' essential extension of $A$ if we show that $\alpha$ is not a proper extension, that is, $\operatorname{dom} \alpha \subseteq A$.

Now $\alpha \pi$ induces a partial homomorphism from $B$ into $A$ such that

$$
\alpha \pi b=\alpha(-\phi b)=-\phi b \quad(b \in \operatorname{dom} \phi) .
$$

Since $\phi$ is irreducible, $B$, dom $\alpha \pi \subseteq$ dom $\phi$, by 0.3 . Let $d \epsilon$ $\operatorname{dom} \alpha$, then $d=a+\pi b$ where $a \in A$ and $b \in B$. Moreover, $\pi b \in \operatorname{dom} \alpha$ since $A \leqslant \operatorname{dom} \alpha$. Therefore $b \in B, \pi^{-1} \operatorname{dom} \alpha=$ Bndom $\alpha \pi \subseteq$ dom $\phi$, and so $\pi b=-\phi b \in A$. Hence dom $\alpha \subseteq$ A.

Finally, let $\psi$ be a partial homomorphism from $A(\phi)$ into A such that $A \subseteq \operatorname{ker} \psi$. Then $\psi \pi$ induces a partial homomorphism from $B$ into $A$ and $\psi \pi(\operatorname{dom} \phi)=\psi(-\phi(\operatorname{dom} \phi)) \subseteq \psi A=0$. Therefore $\operatorname{im} \psi \pi=0$ as $\operatorname{dom} \phi \leq B(A)$. But $A(\phi)=A+\pi B$ and so $\operatorname{im} \psi=\psi A+\operatorname{im} \psi \pi=0$. Thus $A \leq A(\phi)(A)$.

.THEOREM 4.2. If $M$ is a maximal rational extension of $C$, then every rational extension of $C$ is isomorphic over $C$ to 
exactly one submodule of $\mathrm{M}$. If $\mathrm{B}_{1}$ and $\mathrm{B}_{2}$ are two such submodules, then $B_{1} \rightarrow B_{2}$ if and only if $B_{1} \subseteq B_{2}$.

Proof. Let $C \leq B(B)$. Since $C \subseteq \operatorname{dom} \sigma_{B, M} \subseteq B$, $\operatorname{dom} \sigma_{B, M} \leqslant B(B)$ so that $\operatorname{dom} \sigma_{B, M} \leqslant B(M)$ by 2.1. Thus $\sigma_{B, M} \in \operatorname{Fr}(B, M)$ and so by 4.1 is a full homomorphism. Since it is also an isomorphism by $2.4, B$ is isomorphic over $C$ to its image under $\sigma_{B, M}$.

If $C \subseteq B_{i} \subseteq M(i=1,2)$ and $B_{1} \rightarrow B_{2}$, then $\sigma_{B_{1}}, B_{2}$ is a full isomorphism. It extends the identity mapping of $C$ which can be uniquely extended to the identity mapping of $M$. Therefore $\sigma_{B_{1}, B_{2}}$ is the inclusion mapping of $B_{1}$ into $B_{2}$.

In particular, if $B_{1}$ and $B_{2}$ are isomorphic over $C$ we have $\mathrm{B}_{1} \cdot \mathrm{B}_{2}$.

COROLLARY 4.3. The maximal rational extension of $\mathrm{C}$ is unique up to isomorphism over $C$.

As in section 1 , let $I$ denote the ring obtained from $R$ by formally adjoining the ring of integers. There is a natural isomorphism $\mu$ between $C$ and $\operatorname{Hom}(I, C)$ such that

$$
\begin{aligned}
(\mu c) i & =c i & & (c \in C, i \in I) \\
\mu-1 \phi & =\phi 1 & & (\phi \in \operatorname{Hom}(I, C)) .
\end{aligned}
$$

We may identify $C$ with $\operatorname{Hom}(I, C)$, which in turn may be identified with a submodule of $M=\operatorname{Hom}(I, M)=\operatorname{Fr}(I, M)$, where $M$ is some maximal rational extension of $C$. By $3.4, F r(I, M) \approx F r(I, C)$ and it is easily verified that the isomorphism leaves $C=\operatorname{Hom}(I, C)$ elementwise invariant. Hence $M$ is isomorphic over $C$ with $\operatorname{Fr}(\mathrm{I}, \mathrm{C})$. We have thus established

PROPOSITION 4.4. $\operatorname{Fr}(\mathrm{I}, \mathrm{C})$ is a maximal rational extension of $\mathrm{C}$.

If $R$ contains a unity and we confine ourselves to the category of unitary modules, we can replace $I$ by $R$ in the above argument. Hence a unitary module $\mathrm{C}$ has the maximal rational extension $\operatorname{Fr}(R, C)$ relative to the category of unitary modules. Now for unitary $C, \operatorname{Fr}(I, C)$ is also unitary and hence $\operatorname{Fr}(R, C)$ is also the maximal rational extension of $C$ with respect to the category of all modules. 
Again, let $R$ be an algebra over a field $F$ and suppose we study the category of all representation modules of $R$ and $R-F-$ homomorphisms. 4.4 would still hold with respect to this category provided $I$ is obtained from $R$ by formally adjoining the field $F$ in place of the ring of integers.

5. Rirgs of Quotients. A ring $S$ is called an extension of a ring $R$ if there is given an isomorphism of $R$ into $S$. There is no harm in assuming that $S$ contains $R$ as a subring. $S$ can then be regarded as a right $R$-module $S_{R}$, a left $R$-module $R S$ and a two-sided $\mathrm{R}$-module $\mathrm{R}^{\mathrm{S}_{\mathrm{R}}}$. Following Utumi we call an extension $S$ of $R$ a ring of right quotients of $R$ if $S_{R}$ is a rational extension of $R_{R}$. We denote this situation by the formula $R \leq S\left(S_{R}\right)$.

PROPOSITION 5.I. If $S$ and $T$ are rings of right quotients of $R$ then any homomorphism over $R$ of $S_{R}$ into $T_{R}$ is a ring homomorphism and conversely.

Proof. Let $\phi$ be a homomorphism of $S_{R}$ into $T_{R}$ over $R$ and let $s^{\prime}$ be an element of $S$. Define $\psi: S_{R} \rightarrow T_{R}$ by

$$
\psi s=\left(\phi s^{\prime}\right)(\phi s)-\phi\left(s^{\prime} s\right) \quad(s \in S) \text {. }
$$

For any $x \in R$ we have $\phi T=r$, hence

$$
\psi r=\left(\phi s^{\prime}\right) r-\phi\left(s^{\prime} r\right)=0
$$

But $R \leqslant S\left(T_{R}\right)$ by 2.1 ; therefore $\operatorname{im} \psi=0$, so that $\phi$ is a ring homomorphism. The converse is obvious.

PROPOSITION 5.2. If $A_{R} \leqq B_{R}$, than $\operatorname{Fr}\left(B_{R}, A_{R}\right)$ is a ring containing $\operatorname{Hom}\left(B_{R}, A_{R}\right)$ as a subring.

Proof. If $\phi, \psi \in \operatorname{Fr}(B, A)$ the composite mapping $\phi \psi$ is a fractional homomorphism from $B$ into $A$. For dom $\phi \leqslant$ $B(A)$, hence by 1.2

$$
\operatorname{dom} \phi \psi=\psi^{-1} \operatorname{dom} \phi \leqslant \operatorname{dom} \psi \leqslant B(A) .
$$

Therefore $\phi \psi$ can be extended to a unique element of $\operatorname{Fr}(B, A)$, also denoted by $\phi \psi$. It is easy to verify that $\operatorname{Fr}(B, A)$ forms a ring with respect to this definition of multiplication. If dom $\phi$ $=\operatorname{dom} \psi=B$ then $\operatorname{dom} \phi \psi=B$ hence $\operatorname{Hom}(B, A)$ is a subring. 
THEOREM 5.3. Any ring $R$ has a maximal ring of $r i g h t$ quotients $Q$ which is unique up to isomorphism over $R$. Moreover, $Q_{R}$ is the maximal rational extension of $R_{R}$ and every ring of right quotients of $R$ is isomorphic over $R$ to a unique subring of $Q$.

Proof. Let $Q=\operatorname{Fr}\left(I_{R}, R_{R}\right)$. By 5.2 this is a ring extension of $\operatorname{Hom}\left(I_{R}, R_{R}\right)$, which may be identified with $R$, since the natural isomorphism between them is a ring isomorphism. [Q can be regarded as a right $R$-module because it contains $R$ and also in view of the definition

$$
(q \cdot r) i=q(r i) \quad\left(q \in \operatorname{Fr}\left(I_{R}, R_{R}\right), r \in R, r i \in \operatorname{dom} q\right) \text {, }
$$

which is implied by 3.2 . It is easily verified that the se two ways of making $Q$ an $R$-module are in fact the same.] The remainder of the theorem follows from 4.2 and 5.1 .

PROPOSITION 5.4. Given $A_{S}$, if $R \leq S\left(A_{R}\right)$, then any irreducible partial $R$-homomorphis $m$ from any right $S$-module into $A_{S}$ is a partial $S$-homomorphism.

Proof. Given $B_{S}$, let $\phi$ be an irreducible partial homomorphism from $B_{R}$ into $A_{R}$. For any element $d \in$ dom $\phi$ consider the set $F_{d}=\{s \in S \mid d s \in$ dom $\phi\}$. Clearly $F_{d}$ is an $R$-submodule of $S$ and contains $R$. Define $\psi: F_{d} \rightarrow A$ by

$$
\psi s=\phi(d s)-(\phi d) s \quad\left(s \in F_{d}\right) \text {. }
$$

Then $\psi R=0$. Now $R \leq S(A R)$; hence $i m \psi=0$, so that $\phi(d s)=$ ( $\phi d) s \quad\left(s \in F_{d}\right.$ ). Thus $\phi$ coincides with the homomorphism $\phi$ ': $\mathrm{dS} \rightarrow$ A defined by $\phi^{\prime}(\mathrm{d} s)=(\phi d) s$ on the intersection of their domains. By 0.3, $\phi$ extends $\phi^{\prime}$ and so dS $\subseteq$ dom $\phi$, that is $F_{d}=S$. Thus $\phi(d s)=(\phi d) s$ for all $s \in S$. Since this holds for any $\mathrm{d} \in \operatorname{dom} \phi, \phi$ is an $\mathrm{S}$-homomorphism.

PROPOSITION 5.5. Given $A_{S}$, if $R \leq S\left(A_{R}\right)$ then $C \leqslant$ $B$ (AS) if and only if $C \leqslant B$ (A.R), for any $B_{S}$ and $C_{S}$.

Proof. Assume $C \leqslant B\left(A_{S}\right)$ and let $\phi$ be a partial homomorphism of $B_{R}$ into $A_{R}$ such that $C \subseteq \operatorname{ker} \phi$. We want to prove that im $\phi=0$. Without loss of generality we may assume that $\phi$ is irreducible. By $5.4 \phi$ is a partial $\mathrm{S}$-homomorphism. Now $C \subseteq \operatorname{ker} \phi$ and $C \leqslant B\left(A_{S}\right)$ hence im $\phi=0$.

The converse is trivial. 
PROPOSITION 5.6. If $Q$ is a maximal ring of right quotients of $R$ then $Q$ is its own maximal ring of right quotients.

Proof. It suffices to show that any irreducible fractional homomorphism $\phi$ from any $B_{Q}$ into $Q_{Q}$ is full. Now $\phi$ is an $R$-homomorphism and, since $\operatorname{dom} \phi \leqslant B\left(Q_{Q}\right)$, we have $\operatorname{dom} \phi$ $\leq B\left(Q_{R}\right)$ by 5.5. Thus $\phi$ is a fractional homomorphism from $B_{R}$ into $Q_{R}$ which may be extended to a full homomorphism $\phi^{\prime}$ since $Q_{R}$ is rationally complete. Finally, by $5.4, \phi^{\prime}$ is a $Q$ homomorphism and $\phi^{\prime}=\phi$ since $\phi$ is irreducible.

PROPOSITION 5.7. If $\mathrm{C}_{S} \subseteq B_{S}$ and $R \leq S\left(C_{R}\right)$, then $B_{S}$ is an essential extension of $C_{S}$ if and only if $B_{R}$ is an essential extension of $C_{R}$.

Proof. Let $B_{S}$ be an essential extension of $C_{S}$. The identity mapping $\gamma$ of $\mathrm{C}$ is therefore an irreducible $\mathrm{S}$-homomorphism. By 5.4 , its irreducible extension as a partial $R$-homomorphism from $B$ into $C$ is also an $\mathrm{S}$-homomorphism and must therefore be $\gamma$ itself. Hence $\gamma$ is also irreducible as an R-homomorphism, and so $B_{R}$ is an essential extension of $C_{R}$.

The converse is obvious.

PROPOSITION 5.8. If $S$ is a ring of right quotients of $R$ and $T$ is a ring of right quotients of $S$, then $T$ is a ring of right quotients of $R$.

Proof. We are given that $R \leq S\left(S_{R}\right)$ and $S \leq T\left(T_{S}\right)$. By 2.2, $T_{S}$ is an essential extension of $S_{S}$ and $S \leq T\left(S_{S}\right)$. By 5.7 , $T_{R}$ is an essential extension of $S_{R}$ and, by $5.5, S \leqslant T\left(S_{R}\right)$. Again by $2.2, S \leqslant T\left(T_{R}\right)$, and so, by $2.3, R \leq T\left(T_{R}\right)$.

6. Rings with zero left annihilator. We consider a ring $R$ such that $r R=\overline{0 \text { implies } r}=\overline{0 \text { for all } r} \in R$. These are the rings studied by Utumi [12].

PROPOSITION 6.1. If $R$ has zero left annihilator then $\operatorname{Fr}\left(R_{R}, R_{R}\right)$ is a maximal ring of right quotients of $R$.

Proof. We first show that $R \leq I\left(R_{R}\right)$, where $I$ is obtained from $\mathrm{R}$ by formal adjunction of integers. Let $\phi$ be any partial homomorphism from I into $R$ such that $R \subseteq \operatorname{ker} \phi$. Then $(\operatorname{im} \phi) R$ $\subseteq \phi R=0$, hence $\operatorname{im} \phi=0$.

Thus $R \leq I\left(R_{R}\right)$ and by 3.3 there is a canonical isomorphism between $\operatorname{Fr}(I, R)$ and $\operatorname{Fr}(R, R)$ which sends $\operatorname{Hom}(I, R)$ into $\operatorname{Hom}(R, R)$. The image of $\operatorname{Hom}(I, R)$ consists of the endomorphisms of $R_{R}$ induced by multiplication. We may identify this image with $R$; then $\operatorname{Fr}(R, R)$ becomes a maximal rational extension of $R$.

PROPOSITION 6.2. The maximal ring of right quotients contains a unity if and only if $R$ has zero left annihilator.

Proof. If $R$ has zero left annihilator its ring of right quotients $\operatorname{Fr}(R, R)$ contains the identity automorphism of $R$ which is a unity. 
Conversely, assume that $R \leq Q\left(Q_{R}\right)$ and that $Q$ contains an element $q_{0}$ such that $r q_{0}=r$ for all $r \in R$. If $r R=0$ then $r$ induces a partial endomorphism of $Q_{R}$ whose kernel contains $R$. Hence $r Q=0$ and so $r=r q_{0}=0$.

PROPOSITION 6.3. Let $Q$ be the maximal ring of $r$ ight quotients of a ring $R$ with zero left annihilator and assume $A \leq$ $Q\left(Q_{R}\right)$. Then for each homomorphism $\phi: A_{R} \rightarrow Q_{R}$ there exists a unique element $q$ such that $\phi a=q a(a \in A)$.

Proof. $\phi$, regarded as a partial endomorphism of $Q_{R}$, is fractional and so can be extended to a full endomorphism $\phi$ ' of $Q_{R}$ since $Q_{R}$ is rationally complete. Moreover, by $5.4, \phi^{\prime}$ is also an endomorphism of $Q_{Q}$. Therefore, setting $q=\phi^{\prime} 1$, we find

$\phi^{a}=\phi^{\prime} a=\phi^{\prime}(1 a)=\left(\phi^{\prime} 1\right) a=q a \quad(a \in A)$.

PROPOSITION 6.4. Let $A$ be a two-sided ideal in a ring $R$ with zero left annihilator. Then $A \leq R\left(R_{R}\right)$ if and only if $A$ has zero left annihilator in $R$, that is, $r A=0$ implies $r=0$ for all $r \in R$.

Proof. Assume $A \leqslant R\left(R_{R}\right)$ and $r A=0$. Then $r$ induces an endomorphism of $R_{R}$ whose kernel contains $A$. Hence $r R=0$, and therefore $r=0$.

Conversely, suppose $A$ has zero left annihilator, and let $\phi$ be a partial endomorphism of $R_{R}$ such that $A \subseteq \operatorname{ker} \phi$. For any $d \in \operatorname{dom} \phi,(\phi d) A=\phi(d A) \subseteq \phi A=0$ so that $\phi d=0$. Thus $A \leqslant R\left(R_{R}\right)$.

7. Commutative rings. Prior to studying commutative rings we establish the following, due originally to Utumi $[12,(1.3)]$.

PROPOSITION 7.1. If $S$ is a ring of right quotients of $R$, then an element of $S$ lies in the centre of $S$ if and only if it commutes with all elements of $R$.

Proof. Given an element $s^{\prime}$ of $S$ assume $s^{\prime} r=r s^{\prime}$ for all $\mathbf{r} \in \mathrm{R}$. Consider the mapping $\phi$ defined by

$$
\phi s=s^{\prime} s-s^{\prime} \quad(s \in S) \text {. }
$$

It is easily verified that $\phi$ is an endomorphism of $S_{R}$ whose 
kernel contains $R$. Therefore $\operatorname{im} \phi=0$, that is, s's = ss' for all $s \in S$.

PROPOSITION 7.2. If $S$ is a ring of right quotients of $R$, then $S$ is commutative if and only if $R$ is.

Proof. Assume $R$ is commutative; then by 7.1 every element of $R$ lies in the centre of $S$. Hence, again by 7.1 every element of $\mathrm{S}$ lies in the centre of $\mathrm{S}$.

It is easily seen that any anti-automorphism of a ring $R$ can be extended to an anti-isomorphism between its maximal ring of right quotients and its maximal ring of left quotients. If $R$ is commutative the identity automorphism of $R$ is an antiautomorphism. It can be extended to an isomorphism between the two rings of quotients since each is commutative. It follows from this, if not more directly, that any ring of right quotients of a commutative ring is also a ring of left quotients. Hence we may omit any reference to right and left.

PROPOSITION 7.3. If $Q$ is the maximal ring of quotients of a commutative $r$ ing $R$ with zero annihilator, then any element of $R$ has an inverse in $Q$ if and only if it has a zero annihilator in $R$.

Proof. Let $r^{\prime}$ be an element of $R$ having zero annihilator in $R$ and define a homomorphism $\phi$ of $\left(r^{\prime} R\right)_{R}$ into $Q_{R}$ by $\phi\left(r^{\prime} r\right)=r(r \in R)$. This makes sense since $r^{\prime} r=0$ implies $r=0$. Now $r^{\prime} R$ is a (two-sided) ideal of $R$ with zero annihilator since $r r^{\prime} R=0$ implies $r^{\prime}=0$ and hence $r=0$. Therefore $r^{\prime} R$

$\leq Q\left(Q_{R}\right)$ by 6.4. It follows from 6.3 that there exists an element $q \in Q$ such that $\phi\left(r^{\prime} r\right)=q r^{\prime} r$ for all $r \in R$. That is, for all $r \in R, q^{\prime} r=r=q_{0} r$, where $q_{c}$ is the unity of $Q$. Thus $q r^{\prime}-q_{0}$ induces a partial endomorphism of $Q_{R}$ whose kernel contains $R$, and so $\left(q r^{\prime}-q_{0}\right) Q=0$. Hence $q r^{\prime}-q_{0}=\left(q r^{\prime}-q_{0}\right) q_{0}$ $=0$, that is, $r^{\prime}$ has the inverse $q$.

The converse is obvious.

As an immediate consequence we have:

PROPOSITION 7.4. If $Q$ is the maximal ring of quotients of a commutative $r$ ing $R$ with unity, then the elements $r^{\prime} r^{-1}$ $\left(r^{\prime}, r \in R, r\right.$ with zero annihilator in $R$ ) form a subring $F$ of $Q$ which contains $R$. 
This subring $F$ is also a ring of quotients in our sense and it has been called the full ring of quotients $[11, \mathrm{p} .40]$. If, moreover, every non-zero element of $\mathrm{R}$ has zero annihilator, $R$ is called an integral domain, and its full ring of quotients becomes a field, called the field of quotients of $R$.

PROPOSITION 7.5. If $R$ is an integral domain, its maximal ring of quotients is also its full ring of quotients.

Proof. Let $q \in Q, q \neq 0$. Since $Q_{R}$ is an essential extension of $R_{R}$, by 2.2, $q R \cap R \neq 0$, hence there exist $r, r^{\prime} \in R$ with $q r=r^{\prime} \neq 0$. By $7.3, r$ has an inverse $r^{-1}$, so that $q=r^{\prime} r^{-1}$. Thus $Q=F$.

It is not true in general that the maximal ring of quotients of a commutative ring with unity coincides with its full ring of quotients. In section 9 we shall exhibit a ring which is its own full ring of quotients but which is not rationally complete.

The following remark establishes a connection between the theory of rational extensions of a ring and classical multiplicative ideal theory. An integral domain in which all non-zero ideals are invertible is called a Dedekind ring. Let $R$ be such a ring, $Q$ its ring of quotients. We know by 4.2 that all rational extensions of $R$ are isomorphic over $R$ to $R$-submodules of $Q$. These submodules are precisely the fractional ideals of $R$ whose inverses are integral ideals.

\section{Rings with minimum condition.}

PROPOSITION 8.1. Let $R$ be a ring with the minimum condition on right ideals. Then $R$ contains a smallest right ideal $N$ such that $N \leqslant R\left(R_{R}\right)$.

Proof. Let $N$ be the intersection of all right ideals $A$ of $R$ such that $A \leq R\left(R_{R}\right)$. Because of the minimum condition, $\mathrm{N}$ is already the intersection of a finite number of such ideals. Hence $N \leq R\left(R_{R}\right)$ by 1.3 (iii).

PROPOSITION 8.2. Let $R$ be a ring with a smallest right ideal. $N$ such that $N \leq R\left(R_{R}\right)$. Then any homomorphism of $N_{R}$ into $R_{R}$ is an endomorphism of $N_{R}$, and $N$ is a two-sided ideal.

Proof. Let $\phi: N_{R} \rightarrow R_{R}$. Then $\phi^{-1} N \leqslant N \leqslant R\left(R_{R}\right)$ by 1.2 and 1.3 (ii). Hence $N \subseteq \phi^{-1} N$, that is, im $\phi \subseteq N$. In 
particular, if $\phi$ is the homomorphism induced by an element $r$ of $R$, we have $r N \subseteq N$. Therefore $N$ is a two-sided ideal.

PROPOSITION 8.3. Let $R$ be a ring with zero left annihilator and with a smallest right ideal $N$ such that $N \leqslant R\left(R_{R}\right)$. Then

(i) $\mathrm{N}$ is the smallest two-sided ideal with zero left annihilator in $\mathrm{R}$,

(ii) the ring of endomorphisms of $N_{R}$ is a maximal ring of right quotients of $R$.

Proof. By the definition of $\mathrm{N}$, any two-sided ideal $\mathrm{A}$ contains $N$ if and only if $A \leq R\left(R_{R}\right)$. By 6.4, this is the same as saying that $A$ has zero left annihilator. Since $N$ is two-sided, by 8.2 , (i) follows.

Finally, $\operatorname{Fr}(R, R) \cong \operatorname{Fr}(N, R)$ by 3.3 . If $\phi \in \operatorname{Fr}(N, R)$, then $\operatorname{dom} \phi \leqslant N \leqslant R\left(R_{R}\right)$, hence dom $\phi=N$. Thus $\operatorname{Fr}(N, R)=$ $\operatorname{Hom}(N, R)$, which is $\operatorname{Hom}(N, N)$ by 8.2 . Therefore $\operatorname{Fr}(R, R) \cong$ $\operatorname{Hom}(N, N)$.

9. Algebraic rings. We call a ring $R$ (which is not necessarily commutative) algebraic over a field $F$ if $F$ is a subring of the centre of $R$ and if every element of $R$ satisfies a polynomial equation with coefficients in $F$. For example, a ring of finite matrices over $F$ is algebraic over $F$.

PROPOSITION 9.1. If a ring $R$ is algebraic over a field $F$, then any element $r$ of $R$ possesses a two-sided inverse $r^{-1}$ in $R$, provided its left or right annihilator is zero.

Proof. Let $\mathrm{p}(\mathrm{x})$ be a polynomial of smallest degree such that $p(r)=0$. We may assume that $r \neq 0$, and so $p(x)$ will have degree at least 1 . Write $p(x)=x q(x)-f$, where $f \in F$ and $q(x)$ has smaller degree than $p(x)$. Since $p(x)$ was minimal, $q(r) \neq 0$. If $r$ has zero right annihilator $f=r q(r) \neq 0$, hence $r$ has the right inverse $r^{-1}=q(r) f^{-1}$. Since $q(r) r=r q(r), r^{-1}$ is also a left inverse. For the same reason, the result holds when $r$ has zero left annihilator.

EXAMPLE 9.2. Let $F$ be any field and let $R$ be the ring of all (countably) infinite diagonal matrices over $F$ in which all but a finite number of diagonal elements are equal to each other. Let $\mathrm{N}$ be the $\mathrm{ring}$ of all infinite diagonal matrices 
over $F$ in which all but a finite number of diagonal elements are 0 .

Clearly, every element $r \in R$ has the form

$$
r=f+n \quad(f \in F, n \in N)
$$

Since $n$ is the direct sum of a finite matrix and a zero-matrix, it satisfies a polynomial equation $p(n)=0$. Hence $r$ satisfies $p(r-f)$ $=0$. Thus $R$ is a commutative ring in which every element with zero annihilator has an inverse by 9.1 , that is to say, $R$ is its own full ring of quotients.

$\mathrm{N}$ is an ideal of $\mathrm{R}$. It has elements of the form: an $\mathrm{m} \times \mathrm{m}$ identity matrix plus a zero matrix, for any finite $m$. Hence $N$ has zero annihilator. By $6.4, N \leqslant R\left(R_{R}\right)$. In fact, $N$ is the smallest such ideal. For $N=\dot{\Sigma} \cdot F e_{i}$ where $e_{i}$ is the matrix with 1 in the $\mathrm{i}-$ th diagonal place and zero everywhere else. Now if $\mathrm{A}$ $\leq N\left(R_{R}\right)$ then $A$ is the (weak) direct sum of some of these $F e_{i}$. Suppose $F_{j}$ is not contained in $A$, then $F e_{j} A=0$, contrary to the assumption that $A \leq N \leq R\left(R_{R}\right)$, in view of 6.4. Therefore all $F_{j}$ are contained in $A$, and so $A=N$.

It follows from 8.3 that the maximal ring of quotients of $R$ is the ring of endomorphisms of $N_{R}$. It is easy to see that this is the ring of all infinite diagonal matrices. For if $\phi$ is an endomorphism of $N_{R}$, then $\left(\phi e_{j}\right)\left(F e_{i}\right)=0$ if $i \neq j$, hence $\phi e_{j} \in e_{j} F$, and therefore $\phi$ can be induced by multiplication with a diagonal matrix.

The following example shows that even a finite ring need not be rationally complete.

EXAMPLE 9.3. Let $R$ be the ring of all two by two matrices $\left(\begin{array}{l}\alpha \\ \gamma\end{array}\right)$ over a field $F$, for example a finite field, subject to the condition $\alpha+\gamma=\beta+\delta$. The smallest two-sided ideal $\mathrm{N}$ with zero left annihilator consists of all matrices of the form $\left(\begin{array}{ll}\alpha & \alpha \\ \gamma & y\end{array}\right)$. The maximal ring of right quotients of $\mathrm{R}$ is the ring of all two by two matrices over $F$.

In view of 9.1 , the finite ring constructed above has the property that any element with zero left or right annihilator has an inverse in $R$. Hence the ring of right quotients constructed in [6] is $R$, while the maximal ring of right quotients is larger than R.

McGill University 


\section{CORRECTIONS TO PART I}

p. 78, line 4 from bottom, replace "increasing sequence" by "transfinite increasing sequence".

p. 79, line 3 from bottom, replace " $\phi$ " by " $\psi$ ".

p. 80, after line 5 add: "It is under stood that $C \subseteq B$ and $C^{\prime} \leq B^{\prime}$ and that the isomorphism between $C$ and $C^{\prime}$ is induced by that between $B$ and $B ! "$.

p. 80, line 11, replace "d $\in C$ " by " $\psi d \in \in C$ ".

p. 83, line 13, replace "ascending sequence" by "transfinite ascending sequence".

p. 83, line 15 , replace " $\sigma_{B_{i}, B_{i+1}}$ "by " $\sigma_{B_{i}, B_{j}}(i<j)^{\prime}$, 